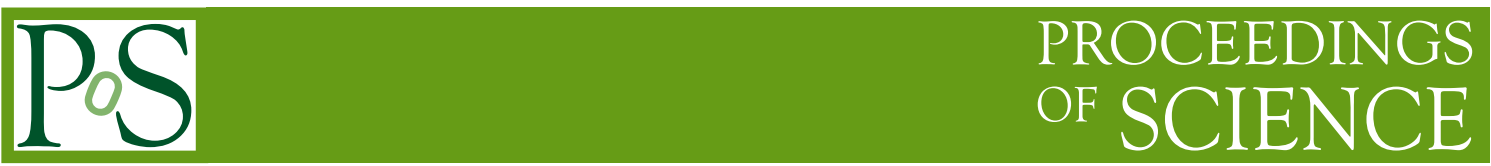

\title{
How to construct a holographic EFT for phonons
}

\author{
Andrea Amoretti* \\ Dipartimento di Fisica, Università di Genova, via Dodecaneso 33, I-16146, Genova, Italy and \\ I.N.F.N. - Sezione di Genova \\ E-mail: andrea.amoretti@ge.infn.it
}

These notes are based on a series of lectures given at the XV Modave Summer School in September, 2019. The course was organized in five lectures on Holographic techniques applied to condensed matter physics. In order to be self consistent, in the first three lectures I introduced the basic concepts of the so called holographic dictionary. Since AdS/CFT is by now a very well established framework to analyze strongly coupled quantum field theories, and many good reviews and books have appeared on the topic, I will refer to this material $[1,2,3,4,5]$ for the introduction to the basic tools requested to understand these notes. What illustrated in here consists instead in the analysis of a holographic model which breaks translation spontaneously or pseudo-spontaneously, the holographic realization of a charge density wave. The model originally appeared in [8], but some of the computations outlined in this manuscript, and in particular the derivation of the Ward Identities, have never appeared in the literature so far. The paper constitute a small exercise which a reader interested in learning more advanced techniques to analyze bottom-up holographic models can use as a warm up to eventually tackle more difficult tasks.

XV Modave Summer School in Mathematical Physics - Modave2019

8-14 September 2019

Modave, Belgium

${ }^{*}$ Speaker. 


\section{Contents}

1. Introduction 1

2. A model for thermodynamically stable phases 2

2.1 Background analysis 3

2.1.1 Background UV asymptotic 3

2.1.2 Background IR asymptotic and thermodynamics 4

2.1.3 Dynamical instabilities of the Reissner-Nordström black hole 5

$\begin{array}{lr}\text { 3. Symmetry pattern and Ward identities } & 7\end{array}$

3.1 The spontaneous case $\phi_{1}=0 \quad 7$

3.1.1 The equilibrium stress-energy tensor $\quad 7$

3.1.2 1-pt Ward identities 9

3.1.3 2-pt Ward identities 9

3.2 The explicit case $\phi_{1} \neq 0 \quad 12$

3.2.1 1-pt Ward identities 13

3.2.2 2-pt Ward identities 13

$\begin{array}{lr}\text { 4. Conclusions } & 15\end{array}$

$\begin{array}{lr}\text { 5. Acknowledgements } & 15\end{array}$

\section{Introduction}

The presence of the ionic lattice in solid materials makes the physical description of condensed matter systems intrinsically non-relativistic. Usually the standard approach to include the effects of the lattice on the electronic plasma is to treat it as an external source of momentum dissipation. Many strongly coupled electronic systems, such as High Temperature superconductors also tends to break translations spontaneously, developing spatial modulations with a periodicity incommensurate to that of the ionic lattice. This ordered phases are commonly called charge density wave (CDW) and spin density wave orders. In this respect, one can imagine that the electronic fluid is breaking translational order spontaneously even though translations are in principle already broken by the presence of the ionic lattice. Eventually, constructing strongly coupled effective field theories to model the behavior of these system is extremely relevant, and it has been one of the leading topic in applied holography in recent years (see e.g. [6, 7, 8, 9, 10, 11, 12, 13, 14]).

At the level of constructing an effective field theory for these system (see e.g. [15]), the standard approach is to couple a charged plasma to a set of scalars which spontaneously acquire a VEV proportional to the space-time coordinates:

$$
\left\langle\Phi_{i}\right\rangle \sim x_{i} .
$$


The holographic model described in these notes mimic exactly this construction, coupling the standard Einstein-Maxwell-Dilaton theory to a set of massless scalar fields $\psi_{i}$ those VEVs will be proportional to the boundary space coordinates $x_{i}$. The main goal of the present manuscript is to explain how to construct thermodynamically stable phases which break translations and to analyze the symmetry pattern of these class of model around their stable vacua.

The notes are organized as follows. In section 2 the basic properties of the system will be described and eventually it will be proven that this model admit a thermodynamically stable phase in which an operator acquires a spatially modulated VEV without having a source. Said otherwise, we will prove that within the range of allowed parameters, the model has a phase in which translations are spontaneously broken. In section 3, using a technique illustrated in [16] we will prove that the Ward Identities associated to the holographic model are exactly the ones expected for a conformal field theory deformed by a scalar operator which can break translations either spontaneously or explicitly. Finally, some concluding remarks can be found in section 4 .

\section{A model for thermodynamically stable phases}

We consider the family of holographic theories:

$$
\begin{aligned}
S=\int d^{4} x \sqrt{-g}\left[R-\frac{1}{2} \partial \phi^{2}-V(\phi)-\frac{1}{4}\left(Z_{1}(\phi)\right.\right. & \left.+\lambda_{1} Z_{2}(\phi) \sum_{i=1}^{2} \partial \psi_{i}^{2}\right) F^{2} \\
& \left.-\frac{1}{2} \sum_{I=1}^{2}\left(Y_{1}(\phi) \partial \psi_{i}^{2}+\lambda_{2} Y_{2}(\phi)\left(\partial \psi_{i}^{2}\right)^{2}\right)\right] .
\end{aligned}
$$

Here there is no implicit summation on $i$ indexes. The equations of motion are

$$
\begin{aligned}
& 0= G_{\mu \nu}-\frac{1}{2}\left(Z_{1}(\phi)+\lambda_{1} Z_{2}(\phi) \sum_{i=1}^{2} \partial \psi_{i}^{2}\right) F_{\mu \rho} F_{v}{ }^{\rho}-\frac{1}{2} \nabla_{\mu} \nabla_{v} \phi \\
&-\frac{1}{2} \sum_{i=1}^{2} \partial_{\mu} \psi_{i} \partial_{\nu} \psi_{i}\left(Y_{1}(\phi)+2 \lambda_{2} Y_{2}(\phi) \partial \psi_{i}^{2}+\frac{\lambda_{1}}{2} Z_{2}(\phi) F^{2}\right) \\
&+\frac{g_{\mu \nu}}{4}\left(2 V(\phi)+\partial \phi^{2}+\frac{1}{2} Z_{1}(\phi) F^{2}+\sum_{i=1}^{2} \partial \psi_{i}^{2}\left(Y_{1}(\phi)+\lambda_{2} Y_{2}(\phi) \partial \psi_{i}^{2}+\frac{\lambda_{1}}{2} Z_{2}(\phi) F^{2}\right)\right) \\
& 0=\nabla_{\mu}\left(\left(Z_{1}(\phi)+\lambda_{1} Z_{2}(\phi) \sum_{i=1}^{2} \partial \psi_{i}^{2}\right) F^{\mu \nu}\right), \\
& 0 \phi-\frac{1}{4}\left(Z_{1}^{\prime}(\phi)+\lambda_{1} Z_{2}^{\prime}(\phi) \sum_{i=1}^{2} \partial \psi_{i}^{2}\right) F^{2}-V^{\prime}(\phi)-\frac{1}{2} \sum_{i=1}^{2}\left(Y_{1}^{\prime}(\phi) \partial \psi_{i}^{2}+\lambda_{2} Y_{2}^{\prime}(\phi)\left(\partial \psi_{i}^{2}\right)^{2}\right), \\
& 0=\nabla_{\mu}\left(\left(Y_{1}(\phi)+\partial \psi_{i}^{2}\left(2 \lambda_{2} Y_{2}(\phi)+\frac{\lambda_{1}}{2} Z_{2}(\phi) F^{2}\right)\right) \nabla^{\mu} \psi_{i}\right), \quad i=1,2 .
\end{aligned}
$$


We will leave the scalar couplings arbitrary, except for specifying their UV $\phi \rightarrow 0$ :

$$
\begin{gathered}
V(\phi) \sim-6+\frac{1}{2} m^{2} \phi^{2}+\ldots, \quad Z_{1}(\phi) \sim 1+z_{2} \phi^{2}+\ldots, \quad Z_{2}(\phi) \sim z_{2} \phi^{2}+\ldots, \\
Y_{1}(\phi)=Y_{2}(\phi) \sim y_{2} \phi^{2}+\ldots
\end{gathered}
$$

and IR $\phi \rightarrow \infty$ asymptotics

$$
\begin{gathered}
V_{I R}=V_{0} e^{-\delta \phi}, \quad Z_{I R}=Z_{0} e^{\gamma \phi}, \quad Y_{I R}=Y_{0} e^{\lambda \phi}, \quad \phi=\kappa \log \phi \\
\lambda_{1 I R}=\tilde{\Lambda}_{1} e^{\tilde{\lambda}_{1} \phi} \quad \lambda_{2 I R}=\tilde{\Lambda}_{2} e^{\tilde{\lambda}_{2} \phi}
\end{gathered}
$$

This ensures that the model remains asymptotically anti-de Sitter in the UV, while the specific form of the metric in the IR encodes the possibility to consider hyperscaling violating geometries (see [8] for more details on the effects of these geometries on the transport properties of this specific model).

\subsection{Background analysis}

Our Ansatz for the background is:

$$
d s^{2}=-D(r) d t^{2}+U(r) d r^{2}+C(r) d \vec{x}^{2}, \quad A=A(r) d t, \quad \phi=\phi(r), \quad \psi_{i}=k \delta_{i j} x^{j} .
$$

\subsubsection{Background UV asymptotic}

From now on we will restrict to the case where $d=2$ and we set the mass squared of the scalar potential to $m^{2}=-2$. The UV expansion of the background (in Fefferman-Graham gauge) reads

$$
\begin{aligned}
D(r) & =\frac{1}{r^{2}}\left(1-\frac{\phi_{1}^{2}}{8} r^{2}+d_{3} r^{3}+O\left(r^{4}\right)\right) \\
U(r) & =\frac{1}{r^{2}} \\
C(r) & =\frac{1}{r^{2}}\left(1-\frac{\phi_{1}^{2}}{8} r^{2}-\left(\frac{d_{3}}{2}+\frac{1}{3} \phi_{1} \phi_{2}\right) r^{3}+O\left(r^{4}\right)\right) \\
\phi(r) & =\phi_{1} r+\phi_{2} r^{2}+O\left(r^{4}\right) \\
A(r) & =\mu-\rho r+O\left(r^{3}\right)
\end{aligned}
$$

where subleading coefficients are fixed in terms of the VEVs $\rho, \phi_{2}$ and $d_{3}$. The asymptotic expansion for the fields $\psi_{i}$ changes if one considers $\phi_{i}=0$ or $\phi_{1} \neq 0$. This is due to the particular UV form of the potential $Y(\phi)$ we have chosen. In fact, in the case $\phi_{1}=0$ the equation of motion (2.5) implies

$$
\psi_{i}=\frac{\psi_{i(-1)}}{r}+\psi_{i(0)}+\mathscr{O}(r)
$$

Eventually, the choice of the background ansatz (2.8) implies that, if $\phi_{1}=0$, we are considering a theory in which the operators $\psi_{i}$ have a VEV which breaks translations while having no source, since the leading term $\frac{\psi_{i(-1)}}{r}$ in (2.10) is set to zero. This is equivalent to have an operator which breaks translations spontaneously. On the other hand, in the case $\phi_{1} \neq 0$, it is easy to see that the UV expansion for $\psi_{i}$ reads:

$$
\psi_{i}=\psi_{i(0)}+\mathscr{O}(r)
$$


which means that the ansatz (2.8) corresponds, in this case, to a theory in which the $\psi_{i}$ operators are breaking translations explicitly with an explicit spatially modulated source $k x_{i}$. In what follows we will analyze separately the two different symmetry breaking patterns by computing explicitly the Ward identities for the theory.

\subsubsection{Background IR asymptotic and thermodynamics}

We are interested in studying finite temperature states, which corresponds to introduce a black hole with a regular horizon at $r=r_{h}$ in the bulk gravitational theory. Eventually the background fields of the theory have the following near horizon behavior:

$$
\begin{aligned}
& d s^{2}=-4 \pi T\left(r_{h}-r\right) d t^{2}+\frac{d t^{2}}{4 \pi T\left(r_{h}-r\right)}+\frac{s}{4 \pi}\left(d x^{2}+d y^{2}\right)+\ldots \\
& A_{t}=A_{h}\left(r_{h}-r\right)+\ldots, \quad \phi=\phi_{h}+\ldots,
\end{aligned}
$$

where $T$ and $s$ are the temperature and the entropy of the black hole respectively, namely:

$$
s=4 \pi C\left(r_{h}\right), \quad T=\left.\frac{1}{4 \pi} \sqrt{-\frac{B^{\prime}(r) D^{\prime}(r)}{B^{2}(r)}}\right|_{r=r_{h}} .
$$

There are two radially conserved quantities in the background equations. The first simply gives the UV charge density and relates it to the electric flux emitted from the horizon

$$
\rho=-\left.\frac{C(r) A^{\prime}(r)}{\sqrt{B D}}\left(Z_{1}(\phi)+2 \lambda_{1} k^{2} \frac{Z_{2}(\phi)}{C}\right)\right|_{r=r_{h}},
$$

The second radially conserved quantity is defined by the relation:

$$
\left[-\rho A(r)+\frac{C^{2}(r)}{\sqrt{U(r) D(r)}}\left(\frac{D(r)}{C(r)}\right)^{\prime}+k^{2} I_{Y_{1}}(r)+2 \lambda_{2} k^{4} I_{Y_{2}}(r)-\lambda_{1} k^{2} I_{Z_{2}}(r)\right]^{\prime}=0,
$$

where

$$
I_{Y_{1}}(r)=\int_{r_{h}}^{r} \sqrt{B D} Y_{1}(\phi), \quad I_{Y_{2}}(r)=\int_{r_{h}}^{r} \frac{\sqrt{B D}}{C} Y_{2}(\phi), \quad I_{Z_{2}}(r)=\int_{r_{h}}^{r} \frac{Z_{2}(\phi) A^{\prime 2}}{\sqrt{B D}} .
$$

In order to express the last identity in terms of the thermodynamical quantities of the theory, we need to compute the renormalized on-shell action and eventually the pressure of the system.

The boundary terms needed to renormalize the background action are:

$$
S_{\text {c.t. }}=\int_{r=\varepsilon} d^{3} x \sqrt{-\gamma}\left(2 K+4+R[\gamma]+\frac{1}{2} \phi^{2}\right),
$$

where $\gamma_{\mu \nu}$ is the induced metric at $r=\varepsilon$ and $K$ is the trace of the extrinsic curvature. The renormalized on-shell action is:

$$
\begin{aligned}
S_{\mathrm{ren}}=\lim _{\varepsilon \rightarrow 0}\left[\int d ^ { 3 } x \left(\sqrt{U(\varepsilon) D(\varepsilon)}\left(\frac{4 C(\varepsilon)}{\sqrt{U(\varepsilon)}}+\frac{C^{\prime}(\varepsilon)}{U(\varepsilon)}+\frac{C(\varepsilon) D^{\prime}(\varepsilon)}{U(\varepsilon) D(\varepsilon)}\right)+\frac{1}{2} \sqrt{D(\varepsilon)} C(\varepsilon) \phi(\varepsilon)^{2}\right.\right. \\
\left.\left.-k^{2} I_{Y_{1}}(\varepsilon)-2 \lambda_{2} k^{4} I_{Y_{2}}(\varepsilon)+\lambda_{1} \rho^{2} k^{2} I_{Z_{2}}(\varepsilon)\right)\right],
\end{aligned}
$$


Evaluating the previous expression on the background (2.9) we obtain:

$$
S_{\text {ren }}=V\left(-k^{2} I_{Y_{1}}(0)-2 \lambda_{2} k^{4} I_{Y_{2}}(0)+\lambda_{1} \rho^{2} k^{2} I_{Z_{2}}(0)+\frac{3 d_{3}}{2}\right)
$$

where $V$ is the boundary volume. Note that the previous expression is independent on the source of the scalar $\phi_{1}$ and holds both in the spontaneous and explicit case. However, $k$ needs to be treated differently in the two cases $\phi_{1}=0$ and $\phi_{1} \neq 0$. In the first scenario, $k$ is a source and can be fixed as a boundary condition of the theory. In this case the pressure is defined as:

$$
P_{\text {exp }}=-\frac{S_{r e n}}{V}=-\frac{3 d_{3}}{2}+k^{2} I_{Y_{1}}(0)+2 \lambda_{2} k^{4} I_{Y_{2}}(0)-\lambda_{1} \rho^{2} k^{2} I_{Z_{2}}(0) .
$$

On the other hand, in the spontaneous case the free energy should be minimized with respect to $k$ to find the most stable phase. This is equivalent to imposing periodic boundary conditions on the spatial coordinates $x^{i}$, with periodicity $L_{x}=2 \pi / k$ (see e.g. [17] for more details). Indeed, this is exactly what we want to describe CDW states. Using (2.19), we get

$$
I_{Y_{1}}(0)+\lambda_{2} k^{2} I_{Y_{2}}(0)-\frac{1}{2} \lambda_{1} I_{Z_{2}}(0)=0,
$$

so that in the end

$$
P_{\text {spont }}=-\frac{S_{r e n}}{V}=\frac{3}{2} d_{3} .
$$

We will see later how this affects the form of the equilibrium stress-energy tensor of the model in the spontaneous and in the explicit case.

Having defined the pressure, upon evaluating the radially conserved quantity (2.15) both at the boundary and at the horizon, one recovers the usual Smarr law:

$$
\mathscr{E}+P=\mu \rho+T s,
$$

where $\mathscr{E}$ is the energy density. The previous expression is valid both in the spontaneous and in the explicit case.

Having described the background geometry, the next question that needs to be addressed is if the spontaneous phase just described exists as the endpoint of instabilities of the (translation invariant) Reissner-Nordström black hole.

\subsubsection{Dynamical instabilities of the Reissner-Nordström black hole}

For simplicity, we require that Reissner-Nordström is a solution of the equations of motion derived from (2.1). To this end, we consider the UV expansion (2.6) for the couplings around $\phi=0$, while in the IR, it becomes an $\mathrm{AdS}_{2} \times \mathrm{R}^{2}$ geometry:

$$
d s^{2}=-\frac{d t^{2}}{\xi^{2}}+\frac{d \xi^{2}}{6 \xi^{2}}+d x^{2}+d y^{2}, \quad \phi=0, \quad A_{t}=\frac{\sqrt{2}}{\xi}, \quad \psi_{i}=0 .
$$

Importantly, $k=0$ (or equivalently $\psi_{i}=0$ ) in the solution (2.24), since we want our starting theory to be invariant under translations.

We now perturb the solution (2.24) with the following radial fluctuations:

$$
\delta \phi=\phi_{0} \xi^{\delta_{\phi}}, \quad \delta \psi_{i}=k \delta_{i j} x^{j} .
$$


The equations of motion for the $\psi_{i}$ 's are automatically satisfied by our Ansatz. As one can see from the equations of motion (2.2)-(2.5), having set $\phi=0$ in the background implies that the radial perturbations involving the scalar decouple from those of the other fields, making the analysis a lot easier. However, there is no conceptual obstacle to repeating this procedure over an $\mathrm{AdS}_{2} \times \mathrm{R}^{2}$ domain-wall with $\phi \neq 0$.

The IR dimension $\delta_{\phi}$ of the operator dual to $\phi$ is easily obtained from the equation of motion for $\phi$

$$
\delta_{\phi}=-\frac{1}{2}+\frac{1}{6} \sqrt{9+6 m^{2}-72 z_{2}+12 k^{2}\left(y_{2}-12 z_{2} \lambda_{1}\right)+12 y_{2} \lambda_{2} k^{4}} .
$$

There is an instability whenever the radicand $\Delta$ changes sign from positive to negative. In order for this instability to be towards a phase with $k \neq 0$ (and so breaking translations spontaneously), we need $\Delta(k)<0$ for $0<k_{-}<k<k_{+}$where $\Delta\left(k_{ \pm}\right)=0$.

It is straightforward to check that this can easily happen in the allowed parameter space on $\lambda_{1,2}$, depending on the specific choice of scalar couplings. The couplings $\lambda_{1,2}$ are constrained by causality: [18] found a necessary condition on $\lambda_{1},-1 / 6<\lambda_{1}<1 / 6$. We take $\lambda_{2}>0$ and defer a more thorough analysis to future work.

For concreteness, we consider a model inspired by [19]:

$$
\begin{gathered}
V(\phi)=-6 \cosh \left(\frac{\phi}{\sqrt{3}}\right), \quad Z_{1}(\phi)=\cosh ^{\gamma / 3}(\sqrt{3} \phi), \quad Z_{2}(\phi)=\frac{1}{2} \gamma \sinh ^{2}(\phi), \\
Y_{1,2}(\phi)=12 \sinh ^{2}\left(\frac{\phi}{\sqrt{3}}\right),
\end{gathered}
$$

for which the regime of dynamical instability is

$$
\begin{gathered}
\gamma<-4, \quad-\frac{1}{6}<\lambda_{1}<\frac{2}{3 \gamma}, \quad 0<\lambda_{2}<-\frac{\left(2-3 \gamma \lambda_{1}\right)^{2}}{(1+12 \gamma)}, \\
\tilde{k}_{-}<\tilde{k}<\tilde{k}_{+}, \quad \tilde{k}_{ \pm}=\frac{1}{2} \sqrt{\frac{3 \gamma \lambda_{1}}{\lambda_{2}}-\frac{2}{\lambda_{2}} \pm \frac{\sqrt{9 \gamma^{2} \lambda_{1}^{2}+12 \gamma \lambda_{2}-12 \gamma \lambda_{1}+\lambda_{2}+4}}{\lambda_{2}}} .
\end{gathered}
$$

It is interesting to note that the new couplings $\lambda_{1,2}$, even for small values, have changed the range of values of $\gamma$ where the dynamical instability lies (which for $\lambda_{1,2}=0$ is $\gamma>-1 / 12$ [19]).

In such a case, we also expect a dynamical instability of the non-zero temperature translationinvariant black hole towards a spatially modulated phase, which can be diagnosed by constructing the corresponding normalizable mode at $k \neq 0$, see e.g. [20] for a concrete example. The outcome of this computation is a so-called 'bell curve' which shows the evolution of the critical temperature below which the condensate forms as a function of $k$. The most stable phase is found for $k_{\star}$ such that $T_{c}\left(k_{\star}\right)$ is maximum.

We now turn to the construction of such a bell curve in our model (2.1) with couplings given by (2.27). This implies constructing the unstable mode at non-zero temperature in the ReissnerNordström black hole background:

$$
\begin{aligned}
& d s^{2}=-r^{2} f(r) d t^{2}+\frac{d r^{2}}{r^{2} f(r)}+r^{2}\left(d x^{2}+d y^{2}\right) \\
& A_{t}=\mu\left(1-\frac{r_{h}}{r}\right), \quad f(r)=1-\frac{r_{h}^{3}}{r^{3}}-\frac{\mu^{2} r_{h}}{4 r^{3}}\left(1-\frac{r_{h}}{r}\right), \quad \phi=\psi_{i}=0 .
\end{aligned}
$$




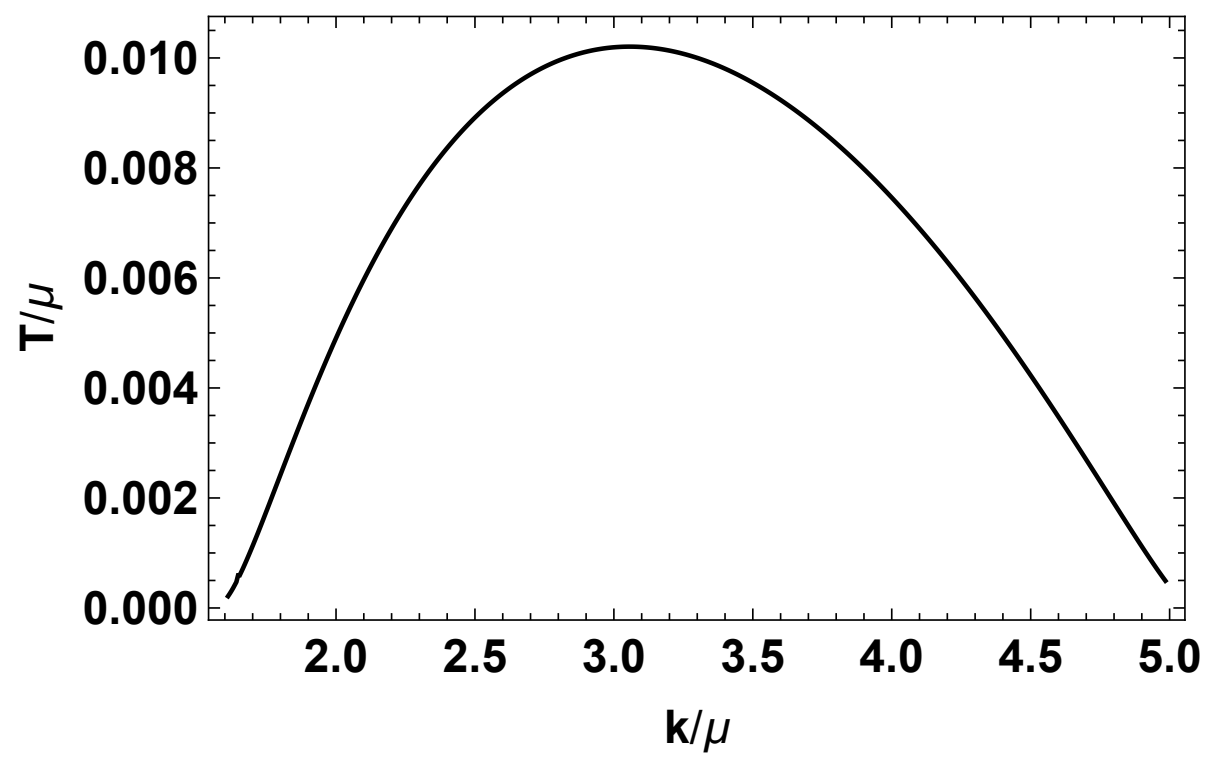

Figure 1: Instability curve $T_{c}(k)$ of the Reissner-Nordström black brane for parameters $\gamma=-6, \lambda_{1}=-0.13$, $\lambda_{2}=5 \cdot 10^{-4}$.

As for zero temperature, the unstable mode obeys a decoupled equation of motion:

$$
\delta \phi^{\prime \prime}+\left(\frac{4}{r}+\frac{f^{\prime}}{f}\right) \delta \phi^{\prime}+\left(-m^{2}-\frac{2 Y_{2} k^{2}}{r^{2}}+\frac{2 r_{h}^{2} \mu^{2} Z_{2} \lambda_{1} k^{2}}{r^{6}}+\frac{-2 k^{4} Y_{2} \lambda_{2}+r_{h}^{2} \mu^{2} Z_{2}}{r^{4}}\right) \frac{\delta \phi}{r^{2} f}=0 .
$$

We impose regularity at the horizon and spontaneous boundary conditions in the UV. We pick values of $\gamma$ and $\lambda_{1,2}$ satisfying (2.28) and find that this mode exists below a certain critical temperature $T_{c}(k)$, see figure 1. $T_{c}(k)$ has the bell shape typical in holography. It peaks at a certain critical value $k_{\star}$, which we expect to be the dynamically preferred value for the backreacted black holes.

\section{Symmetry pattern and Ward identities}

Having constructed a stable vacuum for the theory, we need to analyze the symmetry pattern of the model around this specific vacuum. This can be done by computing the Ward identities, which is the main topic of the following sections. A similar computation applied to a simpler model can be found in [16].

\subsection{The spontaneous case $\phi_{1}=0$}

\subsubsection{The equilibrium stress-energy tensor}

In order to compute the energy density let us work out the linearized on-shell action on the fluctuation. Let us consider the fluctuation of the background fields:

$$
\begin{aligned}
g_{\mu v} & =\bar{g}_{\mu v}(r)+h_{\mu v}\left(x_{M}\right), \\
A_{\mu} & =\bar{A}_{\mu}(r)+\hat{A}_{\mu}\left(x_{M}\right), \\
\phi & =\bar{\phi}(r)+\hat{\phi}\left(x_{M}\right), \\
\psi_{i} & =\bar{\psi}_{i}(r)+\hat{\psi}_{i}\left(x_{M}\right),
\end{aligned}
$$


where the barred fields are the background ones in (2.8), the Latin indexes run other the boundary coordinates while the Greek one other the whole set of bulk coordinates and we have fixed the radial gauge:

$$
h_{r \mu}=\hat{A}_{r}=0 .
$$

Using the background EOMs one can easily verify that the action (2.1), expanded up to linear order in the fluctuations (3.1)-(3.4), reduces to a boundary term:

$$
\begin{aligned}
S_{r e g}^{(1)}=\int_{r=\varepsilon} d^{3} x \sqrt{-g^{b}} & {\left[\nabla_{v} h^{r v}-\nabla^{r} h_{v}^{v}-\delta \phi \partial^{r} \phi^{b}-\left(Y_{1}(\phi)+\frac{\lambda_{1} Z_{2}(\phi)}{2} F^{2}\right) \sum_{i} \delta \psi_{i} \partial^{r} \psi_{i}^{b}\right.} \\
& \left.-2 \lambda_{2} Y_{2}(\phi) \sum_{i} \partial \psi_{i}^{b 2} \delta \psi_{i} \partial^{r} \psi_{i}^{b}-\left(Z_{1}(\phi)+\lambda_{1} Z_{2}(\phi) \partial \psi^{b 2}\right) \delta A_{v} F^{r v}\right],
\end{aligned}
$$

where the covariant derivatives are the ones of the background and the indices are risen and lowered using $\bar{g}_{\mu \nu}$.

It is important to note that, in the case where the source for the scalar $\phi$ is set to zero, $\phi_{1}=0$, the fluctuations assume the following UV boundary expansion:

$$
\begin{aligned}
h_{\mu v} & =\frac{1}{r^{2}}\left(h_{0 \mu v}\left(x_{\mu}\right)+h_{2 \mu v}\left(x_{\mu}\right) r^{2}+h_{3 \mu v}\left(x_{\mu}\right) r^{3}+\ldots\right) \\
\hat{A}_{\mu} & =\hat{A}_{0 \mu}\left(x_{\mu}\right)+\hat{A}_{1 \mu}\left(x_{\mu}\right) r+\ldots \\
\hat{\phi} & =\hat{\phi}_{1}\left(x_{\mu}\right) r+\hat{\phi}\left(x_{\mu}\right) r^{2}+\ldots \\
\hat{\psi}_{i} & =\frac{\hat{\psi}_{-1 i}\left(x_{\mu}\right)}{r}+\hat{\psi}_{0 i}\left(x_{\mu}\right)+\ldots
\end{aligned}
$$

Due to this fact, one need to consider the following set of counterterms in order to renormalize the action:

$$
S_{\text {c.t. }}=\int_{r=\varepsilon} d^{d} x \sqrt{-\gamma}\left(2 K+4+R[\gamma]+\frac{1}{2} \phi^{2}-\frac{1}{2}\left(Y_{1}(\phi)+\frac{\lambda_{1} Z_{2}(\phi)}{2} F^{2}\right) \sum_{i}\left(\psi_{i}-k x_{i}\right)^{2}\right),
$$

Eventually, the renormalized on-shell action evaluated on the fluctuations (3.7)-(3.10) reads:

$$
S_{r e n}^{(1)}=\int d^{3} x\left[\frac{3 d_{3}}{2} h_{0 t t}+\frac{3 d_{3}}{4} h_{0 x x}+\frac{3 d_{3}}{4} h_{0 y y}-\rho A_{0 t}-\phi_{2} \hat{\phi}_{1}\right] .
$$

From the previous relation one can easily compute the expectation value of the stress energy tensor, obtaining:

$$
\begin{aligned}
\left\langle T^{t t}\right\rangle & =\mathscr{E}=-3 d_{3}, \\
\left\langle T^{x x}\right\rangle & =\left\langle T^{y y}\right\rangle=P=-\frac{3 d_{3}}{2}, \\
\left\langle\mathscr{O}_{\phi}\right\rangle & =\varphi_{2}, \\
\left\langle\mathscr{O}_{\psi}\right\rangle & =0 .
\end{aligned}
$$

In this case the stress energy tensor is equivalent to that of an ideal fluid. 


\subsubsection{1-pt Ward identities}

From the form of the stress energy tensor (3.13)-(3.16) one can easily derive the 1-pt Ward identities. In particular, the stress energy tensor is traceless:

$$
\left\langle T_{\mu}^{\mu}\right\rangle=0
$$

implying that dilatations are broken spontaneously. Regarding the 1-pt Ward identity for the translational symmetry, this is trivially satisfied $(0=0)$, due to the isotropy of the background. This is a general feature of this kind of model which breaks translations preserving the homogeneity of the background and is valid also in the explicit $\left(\phi_{1} \neq 0\right)$ case. This is due to the residual shift symmetry $\psi_{i} \rightarrow+\psi_{i}+c$ enjoyed by the action (2.1). In fact, the background ansatz (2.8) breaks both translations and this shift symmetry, but leaves the diagonal group unbroken. Said otherwise, you one can still absorb the effect a translation by shifting the scalars $\psi_{I}$ leaving the background unchanged. This symmetry pattern, which is the same used in [15], is the reason why the background in this class of (axion-like) models does not depend on the space coordinates but translations are broken. Consequently, in order to fully understand the features of the symmetry breaking pattern one needs to analyze the 2-pt Ward identities, as we will do in the next section.

\subsubsection{2-pt Ward identities}

In order to compute the 2-pt Ward identities we need to evaluate the renormalized on-shell action expanded at the second order in the fluctuations fields. Taking into account the boundary fields expansions illustrated in the previous section, it reads:

$$
\begin{aligned}
& S_{r e n}^{(2)}=\int d^{3} x\left[-\frac{3}{4} h_{0 \mu v} h_{3}^{\mu v}+\frac{3}{4} h_{0 \mu}^{\mu} h_{3 \mu}^{\mu}+\frac{3}{2} \bar{g}_{3 \mu \nu}\left(h_{0 \lambda}^{\lambda} h_{0}^{\nu \lambda}-\frac{3}{4} h_{0}^{\mu v} h_{0 \lambda}^{\lambda}\right)\right. \\
&-\frac{3}{4} \bar{g}_{3 \mu}^{\mu}\left(h_{0 v \lambda} h^{0 \nu \lambda}-\frac{1}{2} h_{0 v}^{v} h_{0 \lambda}^{\lambda}\right)-\frac{1}{2} \hat{\phi}_{1} \hat{\phi}_{2}-\frac{1}{2} y_{2} \phi_{2}^{2} \sum_{i} \hat{\psi}_{0 i} \hat{\psi}_{-1 i} \\
&\left.-\frac{1}{2}\left(\hat{A}_{0 \mu} \hat{A}_{1}^{\mu}-\frac{\rho}{2} h_{0 \eta}^{\eta} \hat{A}_{0 t}-\rho \hat{A}_{0 v} h_{0 t}^{v}\right)\right]
\end{aligned}
$$

In order to derive the the Ward identities we need first to use the $z z$ and $\mu z$ components of the Einstein equations to re-express some of the $h_{3 \mu \nu}$ in terms of the sources, namely:

$$
\begin{aligned}
\partial^{v} h_{3 \mu v}-\frac{1}{3} \phi_{2} \hat{\phi}_{1}+\frac{1}{6} y_{2} \varphi_{2}^{2} \sum_{i} k_{i \mu} \hat{\psi}_{-1 i} & +\delta_{t}^{\eta} \frac{\rho}{6}\left(\partial_{v} \hat{A}_{0 \eta}-\partial_{\eta} \hat{A}_{0 v}\right) \\
& -\bar{g}_{3 v \delta} \partial_{\lambda} h_{0}^{\delta \lambda}-\frac{1}{2} \bar{g}_{3 \eta \lambda} \partial_{v} h_{0}^{\eta \lambda}+\frac{1}{2} \bar{g}_{3 v \delta} \partial^{\delta} h_{0 \lambda}^{\lambda}=0 \\
3 h_{3 \mu}^{\mu}- & 3 \bar{g}_{3}^{\mu v} h_{0 \mu \nu}+2 \phi_{2} \hat{\phi}_{1}=0 .
\end{aligned}
$$

In order to implement the previous constraint it is necessary to consider the standard metric decomposition:

$$
h_{0 \mu v}=h_{0 \mu v}^{(t t)}+\partial_{(\mu} h_{0 v)}^{(t)}+\eta_{\mu v} h_{0}+\frac{\partial_{\mu} \partial_{v}}{\square} H_{0}
$$


where $\partial^{\mu} h_{0 \mu \nu}^{(t t)}, h_{0 \mu}^{(t t) \mu}=0$ and $\partial^{\mu} h_{0 \mu}^{(t)}=0$. As a result of the decomposition above we obtain:

$$
\begin{array}{r}
S_{r e n}^{(2)}=\int d^{3} x\left[-\frac{3}{4} h_{0 \mu v}^{(t t)} h_{3}^{(t t) \mu v}+\frac{1}{8} y_{2} \varphi_{2}^{2} \sum_{i} k_{i}^{v}\left(h_{0 v}^{(t)}+\frac{\partial_{v} H_{0}}{\square}\right) \hat{\psi}_{-1 i}-\phi_{2}\left(h_{0}+\frac{1}{2} H_{0}\right) \hat{\phi}_{1}\right. \\
-\frac{1}{2} \hat{\phi}_{1} \hat{\phi}_{2}-\frac{1}{2} y_{2} \phi_{2}^{2} \sum_{i} \hat{\psi}_{i 0} \hat{\psi}_{-1 i}-\frac{1}{2} \hat{A}_{0 \mu} A_{1}^{\mu}-\frac{1}{4} \rho \hat{A}_{0 t}\left(3 h_{0}+H_{0}\right) \\
\left.-\frac{1}{2} \rho \hat{A}_{0 v} h_{0 t}^{v}+\frac{1}{8} \rho\left(h_{0}^{\mu}+\frac{\partial^{\mu} H_{0}}{\square}\right)\left(\partial_{\mu} \hat{A}_{0 t}-\partial_{t} \hat{A}_{0 \mu}\right)\right] .
\end{array}
$$

To work out how the remaining VEVs depends on the sources we need to rely on gauge invariance. In fact, under a general diffeomorphisms plus scale transformation the fields transform as:

$$
\begin{aligned}
& \delta_{\xi+\beta} h_{\mu v}=\partial_{\mu} \xi_{v}+\partial_{v} \xi_{\mu}-2 \beta \eta_{\mu v}, \\
& \delta_{\xi+\beta} \hat{\phi}=\xi_{\mu} \partial^{\mu} \bar{\phi}+\beta r \partial_{r} \bar{\phi}, \\
& \delta_{\xi+\beta} \hat{\psi}_{i}=\xi_{\mu} \partial^{\mu} \bar{\psi}_{i}+\beta r \partial_{r} \bar{\psi}_{i}, \\
& \delta_{\xi+\beta} \hat{A}_{\mu}=\bar{A}_{v} \partial_{\mu} \xi^{v}-\beta \bar{A}_{\mu},
\end{aligned}
$$

where $\xi_{\mu}$ is the vector which parametrize the diffeomorphisms transformation, while $\beta$ is the parameter for the dilatations. Decomposing the vector $\xi$ as $\xi_{\mu}=\xi_{\mu}^{(t)}+\frac{1}{2} \frac{\partial_{\mu}}{\square} \chi$, and taking into account the expansion (3.56) and the decomposition (3.21), we find:

$$
\begin{aligned}
\delta h_{0 \mu}^{(t)} & =2 \xi_{\mu}^{(t)} \\
\delta H_{0} & =\chi \\
\delta h_{0} & =-2 \beta \\
\delta \hat{\phi}_{1} & =0 \\
\delta \hat{\phi}_{2} & =2 \beta \phi_{2} \\
\delta \hat{\psi}_{-1 i} & =0 \\
\delta \hat{\psi}_{i 0} & =k_{i}^{\mu}\left(\xi_{\mu}^{(t)}+\frac{1}{2} \frac{\partial_{\mu}}{\square} \chi\right) \\
\delta A_{0 \mu} & =\bar{A}_{0 v}\left(\partial^{\mu} \xi^{(t) v}+\frac{1}{2} \frac{\partial_{\mu} \partial^{v}}{\square} \chi\right)-\beta \bar{A}_{0 \mu}, \\
\delta A_{1 \mu} & =\bar{A}_{1 v}\left(\partial^{\mu} \xi^{(t) v}+\frac{1}{2} \frac{\partial_{\mu} \partial^{v}}{\square} \chi\right)-\beta \bar{A}_{1 \mu}
\end{aligned}
$$

Eventually, the invariant combinations are:

$$
\begin{aligned}
\Pi_{\hat{\phi}_{1}} & =\hat{\phi}_{1}, \\
\Pi_{\hat{\phi}_{2}} & =\hat{\phi}_{2}+h_{0} \phi_{2}, \\
\Pi_{\hat{\psi}_{-1 i}} & =\hat{\psi}_{-1 i} \\
\Pi_{\hat{\psi}_{i 0}} & =\hat{\psi}_{0}-\frac{k^{\mu}}{2}\left(h_{0 \mu}^{(t)}+\frac{\partial_{\mu} H_{0}}{\square}\right), \\
\Pi_{\hat{A}_{0 \mu}} & =\hat{A}_{0 \mu}-\frac{\bar{A}_{0}^{v}}{2}\left(h_{0 \mu}^{(t)}+\frac{\partial_{\mu} H_{0}}{\square}\right)+\frac{1}{2} h_{0} \bar{A}_{0 \mu}, \\
\Pi_{\hat{A}_{1 \mu}} & =\hat{A}_{1 \mu}-\frac{\bar{A}_{1}^{v}}{2}\left(h_{0 \mu}^{(t)}+\frac{\partial_{\mu} H_{0}}{\square}\right)+\frac{1}{2} h_{0} \bar{A}_{1 \mu},
\end{aligned}
$$


We now express all the components of the metric sources introduced in (3.24)-(3.32) in terms of projectors acting on $h_{0 \mu \nu}$ :

$$
\begin{aligned}
h_{0} & =\frac{1}{2}\left(\eta^{\mu v}-\frac{\partial^{\mu} \partial^{v}}{\square}\right) h_{0 \mu v}, \\
H_{0} & =-\frac{1}{2}\left(\eta^{\mu v}-3 \frac{\partial^{\mu} \partial^{v}}{\square}\right) h_{0 \mu v}, \\
h_{0 \mu}^{(t)} & =2\left(\frac{\partial^{v}}{\square} \delta_{\mu}^{\kappa}-\partial_{\mu} \frac{\partial^{v} \partial^{\kappa}}{\square^{2}}\right) h_{0 v \kappa}, \\
h_{0 \mu \nu}^{(t t)} & =\mathscr{T}_{\mu \nu}^{\alpha \beta} h_{0 \alpha \beta},
\end{aligned}
$$

with

$$
\begin{aligned}
\mathscr{T}_{\mu \nu}^{\alpha \beta}= & \delta_{\mu}^{\alpha} \delta_{v}^{\beta}-2 \partial_{(\mu}\left(\frac{\partial^{\alpha}}{\square} \delta_{v)}^{\beta}-\partial_{v)} \frac{\partial^{\alpha} \partial^{\beta}}{\square^{2}}\right) \\
& -\frac{1}{2} \eta_{\mu v}\left(\eta^{\alpha \beta}-\frac{\partial^{\alpha} \partial^{\beta}}{\square}\right)+\frac{1}{2} \frac{\partial_{\mu} \partial_{v}}{\square}\left(\eta^{\alpha \beta}-3 \frac{\partial^{\alpha} \partial^{\beta}}{\square}\right)
\end{aligned}
$$

Using these expressions, the gauge invariant quantities become

$$
\begin{aligned}
\Pi_{\hat{\phi}_{1}} & =\hat{\phi}_{1}, \\
\Pi_{\hat{\phi}_{2}} & =\hat{\phi}_{2}+\frac{1}{2} \mathscr{P}^{\alpha \beta} h_{0 \alpha \beta} \phi_{2}, \\
\Pi_{\hat{\psi}_{-1 i}} & =\hat{\psi}_{-1 i}, \\
\Pi_{\hat{\psi}_{i 0}} & =\hat{\psi}_{0}-k^{\mu} \mathscr{D}_{\mu}^{\alpha \beta} h_{0 \alpha \beta}, \\
\Pi_{\hat{A}_{0 \mu}} & =\hat{A}_{0 \mu}-\bar{A}_{0}^{v} \mathscr{D}_{v}^{\alpha \beta} h_{0 \alpha \beta}+\frac{1}{2} \mathscr{P}^{\alpha \beta} h_{0 \alpha \beta} \bar{A}_{0 \mu}, \\
\Pi_{\hat{A}_{1 \mu}} & =\hat{A}_{1 \mu}-\bar{A}_{0}^{v} \mathscr{D}_{v}^{\alpha \beta} h_{0 \alpha \beta}+\frac{1}{2} \mathscr{P}^{\alpha \beta} h_{0 \alpha \beta} \bar{A}_{1 \mu},
\end{aligned}
$$

where the projectors $\mathscr{D}_{\mu}^{\alpha \beta}$ and $\mathscr{P}^{\alpha \beta}$ are defined as follows

$$
\mathscr{D}_{\mu}^{\alpha \beta}=\frac{\delta_{\mu}^{\alpha} \partial^{\beta}+\delta_{\mu}^{\beta} \partial^{\alpha}}{2 \square}-\frac{\eta^{\alpha \beta}}{4} \frac{\partial_{\mu}}{\square}-\frac{\partial^{\alpha} \partial^{\beta} \partial_{\mu}}{4 \square^{2}}, \quad \mathscr{P}^{\alpha \beta}=\left(\eta^{\alpha \beta}-\frac{\partial^{\alpha} \partial^{\beta}}{\square}\right)
$$

Gauge invariance implies that

$$
\begin{aligned}
\hat{\psi}_{i 0} & =k_{i}^{\mu} \mathscr{D}_{\mu}^{\alpha \beta} h_{0 \alpha \beta}+a(\partial) \Pi_{\hat{\psi}_{-1 i}}+b_{i}(\partial) \Pi_{\hat{\phi}_{1}}+c_{i}(\partial) \tilde{C}^{j l} k_{j}^{\mu} k_{l}^{v} h_{0 \mu v}^{(t t)}+d(\partial) k_{i}^{\mu} \Pi_{\hat{A}_{0 \mu}}, \\
\hat{\phi}_{2} & =-\frac{1}{2} \mathscr{P}^{\alpha \beta} h_{0 \alpha \beta} \phi_{2}+\sum_{i}\left(e_{i}(\partial) \Pi_{\hat{\psi}_{-1 i}}+l_{i}(\partial) k_{i}^{\mu} \Pi_{\hat{A}_{0 \mu}}\right)+f(\partial) \Pi_{\hat{\phi}_{1}}+g(\partial) \tilde{S}^{j l} k_{j}^{\mu} k_{l}^{v} h_{0 \mu v}^{(t t)}, \\
h_{3 \mu v}^{(t t)} & =m(\partial) h_{0 \mu v}^{(t t)}+\tilde{R}^{i j} k_{i \alpha} k_{j \beta} \mathscr{T}_{\mu v}^{\alpha \beta}\left(\sum_{i} n_{i}(\partial) \Pi_{\hat{\psi}_{-1 i}}+o(\partial) \Pi_{\hat{\phi}_{1}}\right)+p(\partial) T_{\mu v}^{\alpha \beta} \sum_{i} k_{i(\alpha} \Pi_{\left.\hat{A}_{0 \beta}\right)}
\end{aligned}
$$

where the unknown functions of $\partial$ can be determined only by solving the entire model. Plugging the previous expressions in (3.22) one can obtain the form of the correlator. Let us focus, for the 
moment, on the mixed correlators between the stress energy tensor and the scalar operators $\psi$ and $\phi$. They take the form:

$$
\begin{aligned}
& \left\langle T^{\alpha \beta}(x) \mathscr{O}_{\psi_{i}}\left(x^{\prime}\right)\right\rangle=2 i \frac{\delta S_{r e n}^{(2)}}{\delta \hat{\psi}_{-1 i} \delta h_{0 \alpha \beta}}=-\frac{i}{2} y_{2}\left\langle\mathscr{O}_{\phi}\right\rangle^{2} k_{i}^{\mu} \mathscr{D}_{\mu}^{\alpha \beta} \delta^{3}\left(x-x^{\prime}\right) \\
& -i \mathscr{T}_{\mu \nu}^{\alpha \beta} k_{l}^{\mu} k_{j}^{v}\left(\frac{3}{2} \tilde{R}^{l j} n_{i}(\partial)+Y_{0} \mathscr{O}_{\phi}^{2} \tilde{C}^{l j} c_{i}(\partial)\right), \\
& \left\langle T^{\alpha \beta}(x) \mathscr{O}_{\phi}\left(x^{\prime}\right)\right\rangle=2 i \frac{\delta S_{r e n}^{(2)}}{\delta \hat{\phi}_{i} \delta h_{0 \alpha \beta}}=-i\left\langle\mathscr{O}_{\phi}\right\rangle \frac{\partial^{\alpha} \partial^{\beta}}{\square} \delta^{3}\left(x-x^{\prime}\right) \\
& -i \mathscr{T}_{\mu \nu}^{\alpha \beta} k_{l}^{\mu} k_{j}^{v}\left(\frac{3}{2} \tilde{R}^{l j} o(\partial)+\tilde{S}^{l j} g(\partial)\right) .
\end{aligned}
$$

From the previous expressions the Ward identities for the $\psi_{i}$ operator follow directly:

$$
\left\langle\partial_{\alpha} T^{\alpha \beta}(x) \mathscr{O}_{\psi_{i}}\left(x^{\prime}\right)\right\rangle=-\frac{i}{2} y_{2}\left\langle\mathscr{O}_{\phi}\right\rangle^{2} k_{i}^{\beta} \delta^{3}\left(x-x^{\prime}\right), \quad\left\langle T_{\alpha}^{\alpha}(x) \mathscr{O}_{\psi_{i}}\left(x^{\prime}\right)\right\rangle=0 .
$$

To obtain the Ward identity for the $\phi$ operator one needs to implement the following shift:

$$
\left\langle T_{\mu v}\right\rangle_{Q F T}=\left\langle T_{\mu v}\right\rangle+\frac{1}{2} \eta_{\mu v}\left\langle\mathscr{O}_{\phi}\right\rangle
$$

so that:

$$
\left\langle T_{\mu v}(x) \mathscr{O}_{\phi}\left(x^{\prime}\right)\right\rangle_{Q F T}=\left\langle T_{\mu v}(x) \mathscr{O}_{\phi}\left(x^{\prime}\right)\right\rangle+i\left\langle\mathscr{O}_{\phi}\right\rangle \delta^{3}\left(x-x^{\prime}\right) .
$$

The shift is needed to properly define the stress-energy tensor of the dual Quantum Field Theory in the presence of external sources, as explained in [21].

Eventually, the final Ward identities are:

$$
\left\langle T_{\mu}^{\mu}(x) \mathscr{O}_{\phi}\left(x^{\prime}\right)\right\rangle_{Q F T}=2 i\left\langle\mathscr{O}_{\phi}\right\rangle \delta^{3}\left(x-x^{\prime}\right), \quad\left\langle\partial_{\mu} T^{\mu v}(x) \mathscr{O}_{\phi}\left(x^{\prime}\right)\right\rangle_{Q F T}=0
$$

This shows that in the spontaneous case the operator $\psi_{i}$ is responsible for the breaking of translations only, while the operator $\phi$ takes into account the spontaneous breaking of dilatations.

\subsection{The explicit case $\phi_{1} \neq 0$}

In the explicit case the computation proceeds as in the previous sections, however, due to the fact that the background scalar field acquires a source $\phi \sim \phi_{1} z+\phi_{2} z^{2}$, the boundary expansion for the fluctuations is modified as follows:

$$
\begin{aligned}
h_{\mu v} & =\frac{1}{r^{2}}\left(h_{0 \mu v}\left(x_{\mu}\right)+h_{2 \mu v}\left(x_{\mu}\right) r^{2}+h_{3 \mu v}\left(x_{\mu}\right) r^{3}+\ldots\right), \\
\hat{A}_{\mu} & =\hat{A}_{0 \mu}\left(x_{\mu}\right)+\hat{A}_{1 \mu}\left(x_{\mu}\right) r+\ldots \\
\hat{\phi} & =\hat{\phi}_{1}\left(x_{\mu}\right) r+\hat{\phi}\left(x_{\mu}\right) r^{2}+\ldots \\
\hat{\psi}_{i} & =\hat{\psi}_{0 i}\left(x_{\mu}\right)+\hat{\psi}_{1 i}\left(x_{\mu}\right) r+\ldots .
\end{aligned}
$$

This implies that in the explicit case the constant term of $\hat{\psi}_{i}$ is a source instead of a VEV. Eventually, the counterterms needed to renormalize the action are the usual ones expected for the EinsteinMaxwell-Dilaton model (see e.g. [21]):

$$
S_{\text {c.t. }}=\int_{r=\varepsilon} d^{d} x \sqrt{-\gamma}\left[2 K+4+R[\gamma]+\frac{1}{2} \phi^{2}\right],
$$




\subsubsection{1-pt Ward identities}

The renormalized on-shell action linear in the fluctuations reads:

$$
S_{r e n}^{(1)}=\int d^{3} x\left[\frac{1}{2}\left(3 d_{3}+\phi_{1} \phi_{2}\right) h_{0 t t}+\frac{3 d_{3}}{4} h_{0 x x}+\frac{3 d_{3}}{4} h_{0 y y}-\rho A_{0 t}-\phi_{2} \hat{\phi}_{1}\right] .
$$

From the previous relation one can easily compute the expectation value of the stress energy tensor, obtaining:

$$
\begin{aligned}
\left\langle T^{t t}\right\rangle & =\mathscr{E}=-3 d_{3}-\phi_{1} \phi_{2}, \\
\left\langle T^{x x}\right\rangle & =\left\langle T^{y y}\right\rangle=P-k^{2} I_{Y_{1}}(0)-2 \lambda_{2} k^{4} I_{Y_{2}}(0)+\lambda_{1} \rho^{2} k^{2} I_{Z_{2}}(0), \\
\left\langle\mathscr{O}_{\phi}\right\rangle & =\varphi_{2}, \\
\left\langle\mathscr{O}_{\psi}\right\rangle & =0 .
\end{aligned}
$$

Observe that $\left\langle T^{i i}\right\rangle \neq P$, which signals that the dual stress-energy tensor is not that of a fluid. Indeed it is compatible with the equilibrium stress-tensor of an isotropic, conformal crystal [22, 23]:

$$
\left\langle T_{\mathrm{eq}}^{i j}\right\rangle=[p-(G+K) \partial \cdot\langle\Psi\rangle] \delta^{i j}-2 G\left[\partial^{(i}\left\langle\Psi^{j)}\right\rangle-\delta^{i j} \partial \cdot\langle\Psi\rangle\right],
$$

with $K$ and $G$ the bulk and shear moduli respectively. The bulk modulus only contributes to diagonal elements, the shear modulus only to off-diagonal elements. Since our background Ansatz is isotropic, the boundary phonons are simply $\left\langle\Psi^{i}\right\rangle=x^{i}$, and the off-diagonal elements of (3.66) vanish. A linear response analysis is thus needed to determine $G$. Equations $(3.62,3.66)$ lead us to identify the bulk modulus as

$$
K=k^{2} I_{Y_{1}}(0)+2 \lambda_{2} k^{4} I_{Y_{2}}(0)-\lambda_{1} \rho^{2} k^{2} I_{Z_{2}}(0),
$$

which is positive with our definition of the $I$ integrals.

From (3.62), one can read the 1-pt Ward identity for the trace of the stress energy tensor, which assumes the usual form of a theory deformed by a scalar operator:

$$
\langle T\rangle=-\phi_{1} \phi_{2}=-\phi_{1}\left\langle\mathscr{O}_{\phi}\right\rangle
$$

As in the spontaneous case, the 1-pt Ward identity for translation is trivially satisfied due to the homogeneity of the background, and one needs to analyze the 2-pt Ward identities in order to achieve some knowledge about the dynamics of the system.

\subsubsection{2-pt Ward identities}

Due to the differences in the boundary expansions of the fields $\hat{\phi}$ and $\hat{\psi}_{i}$, the quadratic on-shell action is modified as follows:

$$
\begin{array}{r}
S_{r e n}^{(2)}=\int d^{3} x\left[-\frac{3}{4} h_{0 \mu v} h_{3}^{\mu v}+\frac{3}{4} h_{0 \mu}^{\mu} h_{3 \mu}^{\mu}+\frac{3}{2} \bar{g}_{3 \mu v}\left(h_{0 \lambda}^{\lambda} h_{0}^{v \lambda}-\frac{3}{4} h_{0}^{\mu v} h_{0 \lambda}^{\lambda}\right)\right. \\
-\frac{1}{4}\left(3 \bar{g}_{3 \mu}^{\mu}+\phi_{1} \phi_{2}\right)\left(h_{0 v \lambda} h^{0 v \lambda}-\frac{1}{2} h_{0 v}^{v} h_{0 \lambda}^{\lambda}\right)-\frac{1}{2} \hat{\phi}_{1} \hat{\phi}_{2}-\frac{y_{2}}{2} \phi_{1}^{2} \sum_{i} \hat{\psi}_{0 i} \hat{\psi}_{1 i} \\
\left.+\frac{1}{4} \phi_{1} h_{0 \eta}^{\eta} \hat{\phi}_{2}-\frac{1}{2}\left(\hat{A}_{0 \mu} \hat{A}_{1}^{\mu}-\frac{\rho}{2} h_{0 \eta}^{\eta} \hat{A}_{0 t}-\rho \hat{A}_{0 v} h_{0 t}^{v}\right)\right] .
\end{array}
$$


Moreover, the constraints (3.19)-(3.20) acquire corrections due to the presence of a source for $\hat{\phi}$ :

$$
\begin{gathered}
\partial^{v} h_{3 \mu v}-\frac{1}{3} \phi_{2} \hat{\phi}_{1}-\frac{1}{3} \phi_{1} \partial_{v} \hat{\phi}_{2}-\frac{1}{6} \phi_{1}^{2} \sum_{i} k_{i \mu} \hat{\psi}_{1 i}+\delta_{t}^{\eta} \frac{\rho}{6}\left(\partial_{v} \hat{A}_{0 \eta}-\partial_{\eta} \hat{A}_{0 v}\right) \\
-\bar{g}_{3 v \delta} \partial_{\lambda} h_{0}^{\delta \lambda}-\frac{1}{2} \bar{g}_{3 \eta \lambda} \partial_{v} h_{0}^{\eta \lambda}+\frac{1}{2} \bar{g}_{3 v \delta} \partial^{\delta} h_{0 \lambda}^{\lambda}=0, \\
3 h_{3 \mu}^{\mu}-3 \bar{g}_{3}^{\mu v} h_{0 \mu v}+2 \phi_{2} \hat{\phi}_{1}+2 \phi_{1} \hat{\phi}_{2}=0 .
\end{gathered}
$$

The gauge invariant combinations (3.44) remain the same, except the ones for the fields $\psi_{i}$ which are modified as follows:

$$
\begin{aligned}
& \Pi_{\psi_{0 i}}=\hat{\psi}_{0 i}-k^{\mu} \mathscr{D}_{\mu}^{\alpha \beta} h_{0 \alpha \beta}, \\
& \Pi_{\psi_{1 i}}=\hat{\psi}_{1 i},
\end{aligned}
$$

so that requiring gauge invariance implies the following relations between VEVs and sources:

$$
\begin{aligned}
\hat{\psi}_{i 1} & =a(\partial) \Pi_{\hat{\psi}_{0 i}}+b_{i}(\partial) \Pi_{\hat{\phi}_{1}}+c_{i}(\partial) \tilde{C}^{j l} k_{j}^{\mu} k_{l}^{v} h_{0 \mu \nu}^{(t t)}+d(\partial) k_{i}^{\mu} \Pi_{\hat{A}_{0 \mu}}, \\
\hat{\phi}_{2} & =-\frac{1}{2} \mathscr{P}^{\alpha \beta} h_{0 \alpha \beta} \phi_{2}+\sum_{i}\left(e_{i}(\partial) \Pi_{\hat{\psi}_{0 i}}+l_{i}(\partial) k_{i}^{\mu} \Pi_{\hat{A}_{0 \mu}}\right)+f(\partial) \Pi_{\hat{\phi}_{1}}+g(\partial) \tilde{S}^{j l} k_{j}^{\mu} k_{l}^{v} h_{0 \mu v}^{(t t)}, \\
h_{3 \mu \nu}^{(t t)} & =m(\partial) h_{0 \mu \nu}^{(t t)}+\tilde{R}^{i j} k_{i \alpha} k_{j \beta} \mathscr{T}_{\mu \nu}^{\alpha \beta}\left(\sum_{i} n_{i}(\partial) \Pi_{\hat{\psi}_{0 i}}+o(\partial) \Pi_{\hat{\phi}_{1}}\right)+p(\partial) T_{\mu \nu}^{\alpha \beta} \sum_{i} k_{i(\alpha} \Pi_{\left.\hat{A}_{0 \beta}\right)}
\end{aligned}
$$

where the arbitrary functions of $\partial$ should be determined by actually solving the equations of motion.

Eventually, the mixed correlators acquire the following form:

$$
\begin{gathered}
\left\langle T^{\alpha \beta}(x) \mathscr{O}_{\psi_{i}}\left(x^{\prime}\right)\right\rangle=2 i \frac{\delta S_{r e n}^{(2)}}{\delta \hat{\psi}_{0 i} \delta h_{0 \alpha \beta}}=-i k_{l}^{\mu} k_{j}^{v} T_{\mu \nu}^{\alpha \beta}\left(\frac{3}{2} \tilde{R}^{l j} n_{i}(\partial)+\tilde{C}^{l j} c_{i}(\partial)\right) \\
-\frac{i}{2} y_{2} \phi_{1}^{2} k_{i}^{v} \mathscr{D}_{\nu}^{\alpha \beta} a(\partial)-i \phi_{1} \mathscr{P}^{\alpha \beta} e_{i}(\partial) \\
\left\langle T^{\alpha \beta}(x) \mathscr{O}_{\phi}\left(x^{\prime}\right)\right\rangle=2 i \frac{\delta S_{r e n}^{(2)}}{\delta \hat{\phi}_{1} \delta h_{0 \alpha \beta}}=-i \frac{3}{2} \tilde{S}^{l j} k_{l}^{\mu} k_{j}^{v} T_{\mu \nu}^{\alpha \beta} g(\partial) \\
-2 i \phi_{2} \frac{\partial^{\mu} \partial^{v}}{\square} \delta^{3}\left(x-x^{\prime}\right)-i \phi_{1} \mathscr{P}^{\alpha \beta} f(\partial)-\frac{i}{2} \sum_{i} k_{i}^{\mu} y_{2} \phi_{1}^{2} \mathscr{D}_{\mu}^{\alpha \beta} b_{i}(\partial)
\end{gathered}
$$

Finally, the two point Ward identities are:

$$
\begin{gathered}
\left\langle T_{\mu}^{\mu} \mathscr{O}_{\psi_{i}}\right\rangle=-i 2 \phi_{1} e_{i}(\partial), \quad\left\langle\partial_{\mu} T^{\mu v} \mathscr{O}_{\psi_{i}}\right\rangle=-i k_{i}^{v} y_{2} \phi_{1}^{2} a(\partial) \\
\left\langle T_{\mu}^{\mu} \mathscr{O}_{\phi}\right\rangle=-2 i \phi_{2} \delta^{3}\left(x-x^{\prime}\right)-2 i \phi_{1} f(\partial), \quad\left\langle\partial_{\mu} T^{\mu v} \mathscr{O}_{\phi}\right\rangle=-\frac{i}{2} y_{2} \phi_{1}^{2} \sum_{i} k_{i}^{v} b_{i}(\partial)
\end{gathered}
$$

The function $e_{i}(\partial)$ can be adsorbed in the definition of the correlators by using the residual translation+shift symmetry of the system. Finally, using the holographic dictionary and the redefining the stress-energy tensor as in (3.54), one finds:

$$
\left\langle T_{\mu}^{\mu} \mathscr{O}_{\psi_{i}}\right\rangle=0, \quad\left\langle\partial_{\mu} T^{\mu v} \mathscr{O}_{\psi_{i}}\right\rangle=-i k_{i}^{v} \sum_{j}\left\langle\mathscr{O}_{\psi_{j}} \mathscr{O}_{\psi_{j}}\right\rangle
$$




$$
\left\langle T_{\mu}^{\mu} \mathscr{O}_{\phi}\right\rangle=2 i\left\langle\mathscr{O}_{\phi}\right\rangle \delta^{3}\left(x-x^{\prime}\right)-\phi_{1}\left\langle\mathscr{O}_{\phi} \mathscr{O}_{\phi}\right\rangle, \quad\left\langle\partial_{\mu} T^{\mu v} \mathscr{O}_{\phi}\right\rangle=-\sum_{i} k_{i}^{v}\left\langle\mathscr{O}_{\phi} \mathscr{O}_{\psi_{i}}\right\rangle
$$

This shows that the operator $\phi$ remains the only responsible for the breaking of dilatation. However, due to operator mixing, both the operator $\psi$ and $\phi$ couple together in the Ward identity for translations. The mixed correlator $\left\langle\mathscr{O}_{\phi} \mathscr{O}_{\psi_{i}}\right\rangle$ is of order $k^{2}$ so it can be neglected in the low $k$ expansion when computing the scattering rate. However, when the source $\phi_{1}$ is of the same order of $k$ both the modes should be taken into account.

\section{Conclusions}

Having analyzed the stability of the spontaneously broken phase and the ward identities of the model, the reader should by now be convinced that the model presented is a good holographic EFT to describe spontaneous symmetry breaking of translations. The additional shift symmetries of the fields $\psi_{i}$, as already mentioned, is the responsible for the homogeneity of the background and makes the analysis of the model relatively simple, so that the basic properties of the theory can be tested without recurring to intense computational effort. The next step in the analysis would be to actually solve the model and analyze the properties of the correlators and eventually of the transport coefficients of the theory. A detailed discussion on the transport coefficients of the theory can be found in $[8,9,10,12,13,24,25]$. I suggest the interested reader to go trough this papers in order to understand the details of the correlators structure, since, as previously mentioned, I voluntarily kept the discussion in these lecture notes schematic, mainly focusing on the aspects of the model not previously analyzed in the literature.

Finally, it is worth to mention that other holographic models which describe the spontaneous symmetry breaking of translations have been studied in recent years. One of them breaks translations in the dual field theory by introducing a suitable mass term for the graviton in the bulk which breaks diffeomorphisms in a controlled way, the so called massive gravity model (see e.g. $[14,26,27]$ and references therein). The second one introduces translations symmetry breaking considering models with a spatially modulated charge density (see e.g. [6]). It would be interesting to analyze systematically similarities and differences between these two models and the theory presented in these notes.

\section{Acknowledgements}

I am particularly grateful to Riccardo Argurio, Daniel Aréan, Daniel Brattan, Blaise Goutéraux and Daniele Musso for the many discussions on topics related to these notes.

\section{References}

[1] J. Zaanen, Y. W. Sun, Y. Liu and K. Schalm, "Holographic Duality in Condensed Matter Physics".

[2] M. Ammon and J. Erdmenger, "Gauge/gravity duality : Foundations and applications".

[3] S. A. Hartnoll, A. Lucas and S. Sachdev, "Holographic quantum matter," arXiv:1612.07324 [hep-th].

[4] A. Amoretti, A. Braggio, N. Maggiore and N. Magnoli, Adv. Phys. X 2, no. 2, 409 (2017). 
[5] A. Amoretti, "Condensed Matter Applications of AdS/CFT : Focusing on strange metals," doi:10.1007/978-3-319-61875-3.

[6] T. Andrade, M. Baggioli, A. Krikun and N. Poovuttikul, "Pinning of longitudinal phonons in holographic spontaneous helices," JHEP 1802, 085 (2018).

[7] L. Alberte, M. Ammon, A. Jiménez-Alba, M. Baggioli and O. Pujolàs, "Holographic Phonons," Phys. Rev. Lett. 120, no. 17, 171602 (2018).

[8] A. Amoretti, D. Areán, B. Goutéraux and D. Musso, "Effective holographic theory of charge density waves," Phys. Rev. D 97, no. 8, 086017 (2018).

[9] A. Amoretti, D. Areán, B. Goutéraux and D. Musso, "DC resistivity of quantum critical, charge density wave states from gauge-gravity duality,” Phys. Rev. Lett. 120, no. 17, 171603 (2018).

[10] A. Amoretti, D. Areán, B. Goutéraux and D. Musso, "Universal relaxation in a holographic metallic density wave phase,” Phys. Rev. Lett. 123, no. 21, 211602 (2019).

[11] A. Amoretti, A. Braggio, G. Caruso, N. Maggiore and N. Magnoli, "Holography in flat spacetime: 4D theories and electromagnetic duality on the border," JHEP 1404, 142 (2014).

[12] A. Amoretti, D. Areán, B. Goutéraux and D. Musso, "Diffusion and universal relaxation of holographic phonons," JHEP 1910, 068 (2019).

[13] A. Amoretti, D. Areán, B. Goutéraux and D. Musso, "Gapless and gapped holographic phonons," JHEP 2001, 058 (2020).

[14] M. Baggioli and S. Grieninger, "Zoology of solid \& fluid holography — Goldstone modes and phase relaxation,” JHEP 1910, 235 (2019).

[15] A. Nicolis, R. Penco and R. A. Rosen, "Relativistic Fluids, Superfluids, Solids and Supersolids from a Coset Construction,” Phys. Rev. D 89, no. 4, 045002 (2014).

[16] A. Amoretti, D. Areán, R. Argurio, D. Musso and L. A. Pando Zayas, “A holographic perspective on phonons and pseudo-phonons," JHEP 1705, 051 (2017).

[17] A. Donos, J. P. Gauntlett, T. Griffin and V. Ziogas, "Incoherent transport for phases that spontaneously break translations," JHEP 1804, 053 (2018).

[18] B. Goutéraux, E. Kiritsis and W. J. Li, "Effective holographic theories of momentum relaxation and violation of conductivity bound," JHEP 1604, 122 (2016).

[19] A. Donos and J. P. Gauntlett, "Novel metals and insulators from holography," JHEP 1406, 007 (2014).

[20] A. Donos and J. P. Gauntlett, “Holographic striped phases,” JHEP 1108, 140 (2011).

[21] M. Bianchi, D. Z. Freedman and K. Skenderis, "How to go with an RG flow," JHEP 0108, 041 (2001).

[22] P. M. Chaikin and T. C. Lubensky, "Principles of Condensed Matter Physics," Cambridge University Press (1995).

[23] L. V. Delacrétaz, B. Goutéraux, S. A. Hartnoll and A. Karlsson, “Theory of hydrodynamic transport in fluctuating electronic charge density wave states," Phys. Rev. B 96, no. 19, 195128 (2017).

[24] M. Baggioli and A. Buchel, "Holographic Viscoelastic Hydrodynamics,” JHEP 1903, 146 (2019).

[25] A. Amoretti, A. Blasi, G. Caruso, N. Maggiore and N. Magnoli, "Duality and Dimensional Reduction of 5D BF Theory,” Eur. Phys. J. C 73, no. 6, 2461 (2013). 
[26] A. Amoretti, A. Braggio, N. Maggiore, N. Magnoli and D. Musso, "Thermo-electric transport in gauge/gravity models with momentum dissipation,” JHEP 1409, 160 (2014).

[27] A. Amoretti and D. Musso, "Magneto-transport from momentum dissipating holography," JHEP 1509, 094 (2015). 